\title{
Expectativas de la filosofía moral y literatura
}

\author{
MARIA TERESA LÓPEZ DE LA VIEJA DE LA TORRE \\ Universidad de Salamanca
}

Tan lejos como se extienda la aplicación de conceptos a priori se extiende el uso de nuestra facultad de conocer según principios, y con el la Filosofía.

\section{KANT, Critica del jutcio,} Introduccion, II

Motiva subiective moventia. Aun siendo numerosas las razones que han inducido a preguntar de nuevo por la posición que habrfa de ocupar la actividad literaria con respecto a la Filosofía, no resulta del todo sencillo identificar cuál de esas razones ha impulsado de modo más eficaz hacia la actual discusión. Por otra parte, la mayoría de los debates dan la impresión de ir avanzando hacia un punto de difícil retorno, por su complejidad en progresión, y por el elemento agonístico que contienen tantas veces. Tanto cuando derivan hacia un compromiso positivo entre lo literario y lo filosofico como, también, en lo que se refiere a un compromiso negativo; esto es, adverso hacia cualquier forma de intromisión de puntos de vista. Pues implica a conocimientos ya consolidados, normalizados como disciplinas. Por lo general, la Filosofia mantiene una posición ambivalente hacia la interdisciplinariedad y sus consecuencias: Platón y sus expresivas indicaciones sobre el arte permanecen a distancia. Por eso, al replantearse ahora una proximidad, tan estimulante como polemica, entre Filosofía y Literatura, surgen nuevos tópicos a discutir, a la vez que regresan a primer plano teorías influyentes en otro tiempo.

Si bien este estado de cosas redunda por lo general en un notorio beneficio para ambas disciplinas, al menos por lo que concierne a sus cotas de reflexividad y diversificación interna, en este mismo contexto teórico se aprecia, no obstante, una tensión entre los análisis comparativos y ensayos interdisciplinares y, de otro lado, la efectividad de tales avances para el tratamiento de las cuestiones especificas, sustantivas, en cada disciplina. Por ejemplo, ¿en qué sentido contribuye la sensibilidad hacia las formas y tipos de discurso a dilucidar con mayor precisión cuestiones habituales en Filosofía moral, como "bien", «justicia», "deber»? La atención preferente que se viene dedicando desde hace algún tiempo a la Literatura no se entiende, en fin, sin tomar en consideración cuáles son los intereses tcóricos, específicos de la Filosofía actual. Su aproximación al ámbito literario irepresenta un intento de prolongar los recursos que ya estaban a disposición de la racionalidad practica? $¿ \mathrm{O}$ bien suponen un signo de retroceso, en cuanto a autonomía entre formas de conocimiento y los valores que éstas representan? Cualquiera de estos interrogantes se ha convertido en motivo suficicnte para adelantar propuestas de interés. El elenco de autores que han contribuido a ello puede considerarse a estas alturas muy amplio y heterogéneo. Recorrer tales propuestas constituye de por si un objetivo para quien pretenda hacerse cargo del estado de la cuestion. Pero no es lo único que puede hacerse $y$, tal vez, ni siquiera sea lo más interesante. 
En términos muy generales, las perspectivas suelen oscilar entre los imperativos de la reconstrucción o aquellos otros, bien diferentes, que tratan de incorporar la metodologfa procedente de la Crítica literaria.' Los resultados no resultan homogeneos, dada la diversidad de objetivos: la revisión de la Retorica, la autonomla de los discursos, los géneros de la escritura, análisis de recursos específicos como la metáfora, etc. Puesto que, en todo caso, se hace preciso elegir, sugerire aquí que el interés por la Literatura se ha de entender a partir de un cambio de expectativas en torno a la Filosofía moral. Y, en este proceso de seleccion, cabe pensar también que tales expectativas dependen del lugar que ocupen en la construcción de la teoría los motiva subiective moventia, a que Kant se refería. Este punto de vista restringe, ciertamente, la perspectiva amplia que va definiendo poco a poco a la relación Filosofía-Literatura. Sin embargo, ayuda a clarificar algunas de esas expectativas, al menos por lo que se refiere a dos cuestiones de interés: a) los antecedentes más inmediatos, modernos, de la actual discusión -Platón ${ }^{2}$ constituye un antecedente insoslayable, pero menos inmediato-; b) por que la Filosofía, en especial la Filosofía moral, se interesa hoy por la Literatura, aun siendo del todo irreversible el proceso de especialización en los discursos. Motiva subiective moventia representan, posiblemente, uno de los hilos teoricos que conducen en modo directo hasta las expectativas - positivas o negativas-, que la Etica abriga sobre lo literario, como elemento innovador para su propio discurso.

El principal objetivo del artículo consiste en aproximarse a los térninos en los cuales la Literatura puede contribuir indirectamente a clarificar algunos principios de la Filosofía mo- ral. El hilo argumental parte de la definición kantiana de tales principios -principium diudicationis, exsecutionis-, hasta llegar a otras aportaciones recientes. En especial, cuando éstas se concentran sobre el papel que desempeñan los agentes, con sus motivaciones, en la construcción de la moralidad: motiva subiective moventia. Estos ocupan, en principio, un lugar destacado en la discusión que sitúa al discurso filosófico junto a otros tipos de discurso. Pero esta hipótesis depende en gran parte de cómo y hasta dónde se acepten algunas condiciones, centrales para la comprensión de la Ética y de sus cometidos: 1) La subordinación de las tareas de explicación y ejecución -de las acciones, de lo que prescriben las normas - a las tareas de justificacion. 2) Que la disciplina se limite a considerar la Literatura en términos puramente externos. Esto es, tan solo desde su propio punto de vista, sin ánimo de construir una perspectiva suplementaria, mediante la ayuda de otros puntos de vista. 3) De otra parte, téngase en cuenta que la Literatura nunca ha sido un cuerpo inerte, del cual extraer algunos ejemplos o casos prácticos según criterios filosóficos. Como es obvio, la actividad literaria ha ido estableciendo sus propias reglas; ha alcanzado determinados resultados, cuya intencionalidad moral puede ser muy poco significativa en un principio.

\section{I}

Las anteriores condiciones, aquella que se refería a una discusión interna, y la segunda, el tratamiento circunstancial de otra disciplina, tienen que ver en todo caso con el lugar asignado a la subjetividad y sus formas de expresión. Tanto en Filosofía moral como en Literatura. Pero, antes de pasar adclante, parece necesario introdu- 
cir una precision sobre qué cabe esperar de otros tipos de discurso. Digamos que si, de una parte, la Literatura contribuiría - de hecho contribuye algunas veces- en modo positivo a recordar cuán relevantes pueden llegar a ser la variación socio-historica y los motivos individuales, a la hora de formular valores, no obstante, la expectativa de hallar en una disciplina diferente -en este caso la Literatura - una respuesta y una metodología que la Filosofía moderna descalificó de modo bastante explícito, sólo podrá mantenerse de forma restringida. A condición de que también se asuma una parecida tendencia, de exploración y búsqueda, en la actividad literaria; cuyos resultados distan de objetivos tales como presentar una visión del mundo o un modelo para organizar la existencia. El anacronismo de un concepto de Filosofia dogmática sería apenas más llamativo que ese otro anacronismo, consistente en esperar un proyecto formativo procedente de la Literatura.

Esto es: el narrador siguió una trayectoria, tan compleja y crítica como aquella que caracteriza a la Filosofía contemporánea - W. Benjamin se había referido expresamente al declive del papel tradicional, orientador de experiencias, que fue por mucho tiempo la razón de ser de la actividad narrativa-. Y esto estaría arrojando todo el peso acumulado del tiempo sobre pro. yectos, en principio tan verosímiles como pudo serlo alguna vez un programa de "educación estética del hombre». Por tanto, está fuera de lugar un tipo de actitud que consistiría en añorar en lo literario aquello que ya ha dejado de ser una expectativa razonable para la actividad filosofica. Por lo tanto, no se trata de desestructurar, en parte o en su totalidad, a la Filosofía, pensando que la Literatura aún podría ofrecer una visión más centrada de lo real. Un improbable ofrecimiento: sus historias como historias edificantes. 0 como proyectos para orientar en medio de la diversificación que, una y otra vez, atestigua el discurso filosófico. Se trata, por el contrario, de pretensiones más reducidas: reconstituir uno de los principios que han formado parte de la moralidad, pero que había tenido un tratamiento menor en la $\mathrm{Fi}$ losofia moderna - por causas diversas- En especial, cuando ésta se concentra en su calidad de «Filosofía moral». Tras lo cual, intentaré ahora plantear la pregunta del comienzo, ¿por que situar la confluencia de lo filosófico y lo literario en los motiva subiective moventia?

1) En principio, la esfera que corresponde a las motivaciones de la acción ocupa un lugar marginal en las teorias morales de tipo cognitivista. Marginal, pues éstas se concentran en el principio supremo de la moralidad. Pero, incluso en esta función, función básicamente negativa, los motivos informan sobre algo que ha de recordarse de vez en cuando: ponen de manifiesto, siquiera en modo indirecto, al alcance de la Filosofía práctica y sus distintos niveles. Suponen, en fin, reconsiderar un enfoque que habia permanecido en un segundo plano, al menos mientras la tarea fundamental hubo de centrarse casi en exclusiva en el establecimiento de qué sea y cómo se habria de entender la actitud crítica. Según Kant: "Tan lejos como se extienda la aplicación de conceptos a priori se extiende el uso de nuestra facultad de conocer según principios, y con él la Filosofía".

2) Conocimiento y principios a prio$n$ formaban así un marco, al cual habia de ajustarse luego la facultad de desear. Y que hacía tan problemático al sentimiento del gusto. Esto significaba asimismo fijar el ámbito de la re- 
flexión moral del lado de la universalidad; del lado de los principios y, además, de una determinada clase de principios. Por eso, la opción por la universalidad, y por las prerrogativas que compete señalar a la facultad del conocimiento, contribuyó a introducir de paso una jerarqua de niveles: la $\mathrm{Fi}$ losofia moral pura se distingue en adelante de la moralidad que permanece aún en el conocimiento vulgar. Es decir, en su sentido aún cercano a las actitudes, los motivos, los sentimientos. La buena voluntad, el deber señalan, por el contrario, cuál debe ser considerado como el lugar propio de la moralidad. Así quedaba establecido desde la Fundamentacion de la metafísica de las costumbres: a priori, en la razón.

3) ¿Qué hacer entonces con los sentimientos, "el corazon»? ${ }^{3}$ Kant se ocupó fugazmente de esta dimensión antropológica de la moralidad. Para decir que, en todo caso, tiene que ver con la forma de conducir la propia existencia. Pero no era lo primordial; de ahí que, en aras del principio supremo de moralidad, los principios adicionales pasasen a ocupar una posición secundaria. $\mathrm{E}$ incluso fueron rechazados casi en su totalidad, como exponentes de una versión "patológica" de la misma moralidad, tal como se afirmaba en las Lecciones de Filosofia moral. Pues bien, esta toma de postura conducia en efecto a una radical diversificación de principios. Mientras que unos conciernen a la justificación teórica, se hacía necesario arbitrar otros principios para la esfera subjetiva de la acción. Es decir, lo "subjetivo" y "objetivo" de la moralidad ocupaban posiciones tan distantes entre sí, como el entendimiento con respecto a los sentimientos, el «corazón».

4) Principitim exsecutionis, se refería al fundamento subjetivo. Principium diudicationis al fundamento ob- jetivo. El criterio para establecer juicios y, de otro lado, aquello que impulsa a actuar no pertenecen al mismo nivel, ni se han de confundir. Kant advertía también de la superioridad del fundamento objetivo en lo teorico. Por tanto, la reflexión moral se desentendía en buena parte de los móviles e inclinaciones, que podrian hacer efectiva en algún momento a la moralidad. Pues actos y agentes representaban la parte menos pura de ésta, la moralidad. En lo sucesivo, no habrá duda alguna, el principio supremo corresponderá a la razón: como principium moralitatis purum intellectualle intemum.

Un principio "objetivo» e "interno" para la Moral significa que la validez y sus criterios no pueden ser de carácter privado. Ni hallarse vinculados a los sentimientos -como factores inestables, patológicos-, sino a categoricidad que corresponde a la ley moral. De modo que el principio de la moralidad confirma su carácter interno, sin relación ya con los sentimientos o elementos de naturaleza pragmática - confinados desde entonces al ámbito de lo meramente subjetivo, pero no interno-. Así concluye el análisis de los fundamentos, subjetivo y objetivo, de la moralidad. La subordinación de las tareas de explicación y uejecucion» a las tareas de justificación configura, en sus rasgos generales, una noción de juicio moral que no se verá afectado por las inclinaciones. $\mathrm{Ni}$ por todos aquellos factores que mueven a actuar.

\section{II}

Las expectativas en torno a la Filosofia moral no se han modificado de modo sustancial en este aspecto especifico, los motiva subiective moventia. Al menos para aquellos enfoques que han acatado, como punto de referencia, al programa crítico de Kant. Pues éste 
ejemplifica de modo satisfactorio los avances del pensamiento en su etapa moderna. Como éste había seguido una línea de "fundamentación interna", cualquier hipotético acercamiento posterior a otras disciplinas, a disciplinas no filosóficas, vendrá condicionado por el lugar asignado a la perspectiva «externa». De lo que resulta una subordinación clara de las tareas de explicación y "ejecución" - motivos para llevar a acción lo que prescriben las normas - con respecto a las tareas de justificación. $Y$ esta prioridad del juicio sobre los motivos se mantiene hasta llegar a planteamientos recientes de la Filosofía moral. Planteamientos en los que vuelven aquellas o parecidas dificultades, de incipiente presencia en la filosofia kantiana. Por eso, los motivos de la acción apenas se advierten, apenas si resultan significativos para la construcción de la teoría. Sin embargo la situación cambiará, caso de atender al segundo principio de la moralidat. Aparece entonces la hipótesis de una Literatura con capacidad de respuesta ante las restricciones que se ha impuesto a sí misma la Filosofia moral.

Ahora bien, aun suponiendo que la actividad literaria se hubiese mantenido todo este tiempo en una mayor proximidad con respecto a los móviles de la conducta, ¿esta circunstancia sería suficiente para inducir un cambio de marcha en la misma Filosofía? Algunos términos corteses sobre la interdisciplinariedad, como modo de proceder, apenas si ocultan la óptica externalista que adoptan con frecuencia los filosofos, cuando se asoman a la Literatura. La previa aceptación del principio moral -interno, puro e intelectual- no sugiere una actitud distinta al respecto. $\mathrm{Ni}$ una necesidad efectiva de seguir hasta el final a los otros usos de la razón. Algunos pasos importantes se han venido dando en este sentido, restrin- giendo al máximo los posibles intercambios entre Filosofía y Literatura:

a) Por lo general, la posición de J. Habermas sobre la continuidad entre ambos discursos - sobre todo en Nachmetaphysisches Denken o bien Der philosophische Diskurs der Modernemarca la pauta frente a algunas pretensiones irrestrictas de la crítica literaria con respecto al discurso filosófico. Sin embargo, tal vez resulte más ilustrativo aludir a otros aspectos y pasajes de su obra en relación con el tema. Por ejemplo, el conjunto de restricciones que operan para la teoría, de las cuales solo una parte afectaría a la recepción de la Literatura. La tcoría sigue la dinámica de sus problemas propios, más allá de las expectativas desmesuradas, que pretenden obtener de aquella bien una imagen orientadora o la hipotética resolución de cuestiones vitales para el sujeto. En suma, el filósofo no puede tener opinión formada sobre todos los asuntos. La misma noción de "discurso" implicaba poner entre paréntesis a las experiencias y a los contextos de la acción. Por tanto, las cuestiones de génesis quedaban relegadas por las cuestiones de validez. Su negativa a equiparar habla de ficción y habla seria se sitúa, pues, dentro de una opción más general sobre la Filosofía moral; así como sobre los límites de toda teoría y sus pretensiones de validez. De la noción de teoría y de racionalidad derivan, por lo tanto, sus posiciones en torno a las tendencias postvanguardistas, como voluntad de allanar lo que parece diferente. ${ }^{5}$ Las ventajas de un programa de fundamentación racional -o diudicationis, en los términos kantianos- prevalecen sobre otros programas.

b) Otras propuestas, aun permaneciendo en la línea señalada desde la obra kantiana, se interrogan no obstante por los niveles y procesos que 
anteceden y, en cierta forma, motivan la emergencia de la teoria. Tanto $A$. Wellmer como S. Benhabib, por ejemplo, se refieren a esa línea de cuestiones "contingentes", por la que ha de pasar alguna vez la Filosofía moral. A. Wellmer incide en la formación misma del juicio moral, en la dirección de una Etica discursiva que admita el falibilismo. Pues el punto de vista de los fines sub specie aeternitatis descuida lo referente a la mediación entre universal y particular. En efecto, las situaciones suelen ser moralmente complejas, de manera que los sujetos se hallan con frecuencia ante situaciones encontradas. S. Benhabib por su parte no ha dejado de señalar la paradójica ausencia de destinatarios de la Teoría crítica en su última etapa. Pero, más allá de las objeciones a una Ética ahistórica, su propuesta recupera a un yo incardinado en prácticas materiales. Contingencia y circunstancias no sólo no debilitan la conciencia moral postconvencional, sino que constituyen un punto de partida para la moralidad.

Las tensiones entre una "comunidad de derechos" y una "comunidad de necesidades" revela entonces la ficcion epistemologica sobre la cual se había venido asentando el pensamiento moderno. Hacerse cargo ahora de lo que fue eliminado por una estrategia procedimental muy precisa significa recuperar algunos elementos antes ausentes de la teoría, pero activos en las relaciones. Como sugiere P. Bourdieu, tal vez se trata de descubrir o de redescubrir la exterioridad en lo interno o una interiorización de lo externo. En principio, se ha de atender a la diversificación, entre cuestiones que implican justificación teórica $y$, en otro extremo, cuestiones que atañen a la toma de decisiones por parte de los agentes morales. Pero, en un segundo término, no funciona del todo bien la sujección de las tareas de realización -exsecutionis decla Kant- a las tareas de justificación téorica. Al menos en el caso de una moralidad "productiva"; en el sentido de desencadenante de la autoreflexión. En una de sus primeras obras, J. Habermas ${ }^{7}$ atribuía precisamente a ésta un papel singular, de interés emancipatorio.

\section{III}

La Filosofia moderna no siempre ha seguido al pie de la letra aquella recomendación de Descartes, de proceder como «sin ojos ni oídos". Aun habiendo reconocido cuán relevantes podian llegar a ser los motivos e inclinaciones. no obstante éstos suelen permanecer muy por detrás de los afundamentos objetivos" - pero internos- en la configuración global de la Filosofía. En términos más actualizados, esta objetividad supone que no podra sugerirse la autonomía para aquellas propuestas que exhiban su índole "subjetiva". Es decir, si se refieren tan solo a motiva subiective moventia, tal vez externos on cuanto a su genesis. Por ejemplo, el protagonismo creciente de voces alternativas en la construcción de la moralidad no resulta, aún hoy, un argumento suficiente como para modificar la orientación general de la Ética o de sus principios básicos. ¿Por qué? La razon ha de buscarse, como ha recordado H. Dubiel, ${ }^{8}$ entre las tareas que tienen su móvil en la denuncia de las formas de dominio o, por el contrario, en los programas más constructivos, como búsqueda de criterios de emancipación.

Como reacción al abierto predominio de los problemas de argumentación y justificación - mostrando escasamente cuáles habrian de ser sus conexiones con el universo vital-, el contextualismo de R. Rorty ejemplifica 
una actitud positiva hacia lo literario que supone, no obstante, una desregulación completa de las funciones que se atribuyeron durante mucho tiempo a la Filosofía: el textualismo como forma postfilosófica. A. Danto' no pretende llegar a tales consecuencias, si bien atribuye a la perdida de relieve vital de la Filosofía la presencia de la Literatura en el ámbito de aquélla; con el resultado de una cierta recuperación de un programa educativo, un modo de transformar a la audiencia. A. Palmer no acepta que la Literatura aporte tan sólo una ilustración insignificante, periférica de conceptos filosóficos; de la misma manera que $K$. Walton propone considerar en serio a las wverdades de ficciónn. ${ }^{10} \mathrm{M}$. Nussbaum se refiere por su parte a la necesidad de establecer ciertas verdades sobre la vida humana -con sus particularidades y complejidad-, lo cual sólo parece posible en las formas caracterfsticas de la namativa. Ahora bien, al reconducir algunas de estas aportaciones hacia los términos kantianos, expuestos poco antes, se observa mucho mejor la voluntad de aproximar la Filosofía a la actividad literaria. Por qué desplazar las expectativas hacia otros ámbitos del conocimiento.

Parece que la presencia de la Literatura responde, en fin, a una ausencia llamativa en la misma Filosofía moral. A la ausencia de una moralidad sproductiva", por asi decirlo. Lo cual hace pensar en la necesidad de reforzar el papel de los procesos hetrísticos, a fin de llegar a una atención más equilibrada hacia las bases, tanto subjetivas como objetivas de la moralidad. $Y$, en cuanto a los procedimientos, la apelación circunstancial a lo literario res. ponde en su mayor parte a la pretensión de atender, en modo más equilibrado, a los problemas derivados de la aceptabilidad de las normas morales.
Por lo tanto, la Filosofía moral se habria impuesto una aproximacion a otros tipos de discurso, en razón de sus propios intereses y desde sus propias carencias. Sería inconsecuente que esta aproximación procediera ahora en términos puramente externos, de otra parte. La Literatura responde entonces a un conjunto de expectativas bastante reducidas, pero significativas para el momento en que se encuentra la Filosofía práctíca. Por ejemplo, podría indagar a través de la Literatura por su propio potencial explicativo. Para terminar, algunos breves comentarios sobre este último aspecto:

1. ¿Hasta dónde pueden alcanzar las expectativas de la Filosofía moral con respecto a la Literatura? En principio, la actividad literaria suele ser considerada con cierta desconfianza, precisamente por sus rasgos de creciente autonomía en ejercicio. Además porque, con excepción de formas muy rebasadas de naturalismo, la obra literaria no se ajusta en intención a las demandas del filósofo moral y a sus problemas especificos. Por lo general, éste no se mueve con la suficiente soltura entre los avances de la Literatura, resultándole más sencillo formarse una idea de la misma en base a etapas pasadas de la narrativa, ya filtradas por las categorias de la crítica literaria. En este sentido, la Literatura no podría representar un elemento innovador para la reflexión filosófica; por la elemental razón de que aparece plenamente instrumentalizada, como prueba indirecta, y desde luego involuntaria, para una metodologia obsoleta de interpretación y una ideología conservadora. Tal vez, y como sucede con la Filosofía, se espera en vano que la actividad litcraria alcance a neutralizar la vision descentrada de lo real, la autonomía de la esfera moral con respecto a los valores estéticos. Por eso una dis- 
cusión, puesta al día, no debe insistir tanto en diferencias de sesgo epistemológico -las distintas pretensiones de verdad en la Filosofía y la veracidad de la expresividad literaria-, sino en su perspectiva moral.

2. F. Schiller confio en que la libertad seria también accesible desde la belleza. Pensaba que los modernos estaban aquejados de una seria pérdida, la de aquel estado de armonía del que parecian disfrutar los seres humanos en otras épocas. Tal herida, infringida sin remedio al género humano, invitaba precisamente a recuperar la educación de la sensibilidad, en bien de la época ilustrada. Pero, entre tanto, $M$ Weber" hizo ver en todos sus extremos que el proceso de diferenciación y autonomía de las disciplinas apuntaba ya a un estadio diferente de la racionalidad. O mejor dicho, de los tipos de racionalidad, para el orden mundano, secularizado. Por eso, a la vuelta del tiempo, resulta difícil admitir que la Literatura pueda aparecer en el horizonte de la Filosofía moral como garante de un proyecto de integración entre ámbitos. $O$ como lugar privilegiado, parcialmente inmune a la diversificación de la cultura, en el cual habrían permanecido latentes algunas formas. Por el contrario, las formas no aspiran a estructurar la visión de una realidad fragmentaria. Como si fuesen una explicación en clave, o una reserva de programas de acción. Como si fuesen un ejemplo válido para emprender desde la Filosofia aquella sugestiva $-y$ fallida- educación estética de la humanidad.

3. En ocasiones, la actual Filosofía moral parece sometida a una suerte de ansiedad sobre la naturaleza misma de lo moral. ${ }^{12}$ De ahí, la imposibilidad de redescubrir algo, en los términos que fueron empleados con anterioridad. Lo cual apenas si representa una parte del problema. Es decir, del problema consistente en la "presentación sensible" ${ }^{13}$ y su interes. Entonces, si bien R. Barthes, ${ }^{14}$ por ejemplo, rechazaba la hipotesis de que la Literatura haya de mantener una especificidad puramente estética - como si fuera posible un objeto literario en si mismo-, la plena asunción de las dimensiones críticas, y no sólo poéticas, de la escritura tampoco animarta al antiguo proyecto de llegar a la libertad a través de la belleza. De aquel esbozo de integración, de proyecto que afirmaba un sentido, sólo resta su exactitud. "Exactitud" de la propia actividad de escribir, entendida ésta como la capacidad de nombrar los detalles. Literatura, pues, como lenguaje indirecto que, a través de sus técnicas, desmiente toda interpretación instrumental, meramente expresivista del lenguaje: porque no muestra causas o fines, sino su misma precisión. "Endoscópica» incluso, como ya funcionaba en la obra de Proust.

4. La Literatura no describe montanas. Tal vez sólo su recuerdo, desde la conciencia. ${ }^{15}$ En un proceso paralelo, la atención hacia las contingencias $y$ motivos del sujeto reclama su lugar en la Filosofia moral. La tendencia a recuperar el principium exsecutionis puede intepretarse, ciertamente, como incipiente causa de declive para el principium diudicationis. Por el contrario, tan sólo significa que había dos subprogramas en la Filosofía moderna, cuya articulación no ha sido concluida de modo satisfactorio. Motiva subiective moventia indican qué impulsa a actuar, en efecto. Pero a actuar, según los criterios previamente establecidos por el mismo entendimiento. Por tanto, los motivos impulsan hasta donde el entendimiento no habria sido capaz de llegar; sin reclamar para si las funciones de la racionalidad. Motivos y razones corresponden, en suma, a los 
dos fundamentos que Kant propuso para la moralidad, "subjetivo" y "objetivo". El entendimiento ofrecerá el principio supremo y objetivo para el juicio moral, mientras que el principio que mueve hacia la acción moral descansa ken el corazón.

Motiva subiective moventia permiten entender algunas razones por las cuales la Filosofía moral acepta o rechaza, alternativamente, la continuidad con respecto al punto de vista de la Literatura. El lugar central del enfoque epistemológico quedaba sancionado en la iniciativa kantiana, pero - si se me permite también - no anulaba el enfoque antropológico que rescataría a los factores subjetivos. Los sentimientos morales fueron recogidos puntualmen- te, e incluso tenian un sucinto tratamiento en el esquema kantiano. El posterior cambio de expectativas ha significado también una creciente apelación al principio subjetivo de la moralidad. Siquiera en forma de ausencia. Pues, como M. Foucault ${ }^{16}$ recordaba, las prácticas reflexivas no sólo fijan reglas de conducta sino que, de otro lado, contribuyen a la transformación de quienes las asumen. Tal vez se trata tan sólo de acomodar mejor el punto de vista, como respuesta indirecta a las máximas exigencias de la facultad de conocer: "Tan lejos como se extienda la aplicación de conceptos $a$ priori se extiende el uso de nuestra facultad de conocer según principios, y con él la Filosofías.
1. L. Ferry sitúa el origen de las dos tendencias de la Filosofia en momentos distintos, el de la construcción de sistemas y el de su deconstrucción. Este autor se inclina por la posibilidad de un tercer momento, de actualización de la obra kantiana, pues ésta permitina conservar la significación de la subjetividad, así como rectefinir las tareas de la Filosoffa contemporánea. "Kant, penseur de la Modemité, Magrazine Littéraite, 309 (1993), pp. 18-22.

2. I. Murdoch comentaba las objeciones plató. nicas a la función mimetica del arte. Arte que aparece, de un lado, como una forma especial de discemimiento con respecto a la realidad. Por tanto, la actividad artística sirve para comunicar. pan revelar algo; mas de otro lado, representa asimismo un falso conocimiento, por haberle atribuido Platón la capacidad de minar el sentido de lo real. Tal ambiguedad no parece, sin embargo, suficiente como para negarle su dimensión reflexiva y sus efectos educativos. En concreto, I. Murdach se muestra favorable a la presencia del arte en la Filosoffa, aludiendo incluso a la necesi. dad que la disciplina tiene de metaforas. El fuggo y el sol, México, FCE, 1982, pp. 63-86 y 157-160.

3. I. Kant, Vom obersten Prinzip der Moralitätw, en Gesammelte Schriften, Akademie Ausgabe, Berlín, De Gruyter, 1979, vol. XXVI, pp. 1.4221.433 .

4. J. Habermas, Vergarygentheit als Zukunt, Munich, Piper, 1993. pp. 130.158; Wahrheitstheo- rien", en Vorstudien und Ergdnzungen zur Theorie des kommunikativen Handelns. Francfort, Suhrkamp, 1984, pp. 130-131.

5. Ober Titel, Texte und Termine oder Wie man den Zeitgeist rellektiert , en J. Habermas y W. Pehle, Der Autor, der nicht schreibt, Verstuche aber den Buchermacher und das Buch, Francfort, Fischer, 1989, pp. 3-6.

6. A. Wellmer, Elhik und Dialog, Francfort, Suhrkamp, 1986; S. Benbabib, Critique, Norm and Utopia, Nueva York, Columbia University Press, 1986; „Die Modeme und die Aporien dei kritischen Theorie», en W. Bonss y A. Honneth, Sozialforschung als Kritik, Francfort, Suhrkamp, 1982, pp. 127-175.

7. En el texto de Habermas, corrospondiente al an̆o 1965, eErkenntnis und Interessen, luego recogido en Technik and Wissenschaft als aldeologien, Francfort, Suhrkamp, 1968, pp. 146-168.

8. H. Dubiel, «Herschaft oder Emanzipation?, der Streit um die Erbschaft der Kritischen Theoriew, en A. Honneth y Th. McCarthy, Zwischenbetrachrumgen, Francfort, Suhrkamp, 1989, pp. 504-518.

9. R. Rorty, Consequences of Pragnatisn, Minneapolis, University of Minnesota Press, 1982, pp. 139-159; A. Danto: "Philosophy asland/of Literature*, en J. Rajchman y C. West, Post-Analytic Philosoyhy, Nueva York, Columbia University Press, 1985, pp. 63.83.

10. A. Palmer, \&hilosophy and Literature*, Philosophy, 252 (1990); K. Walton, «Fearing Fictions", 
The Joumal of Philosophy (1978), pp. 5-27. Sobre esta perspectiva se puede consultar el comentario de G. Currie: Works of Fiction and Ilocutionary Acts., Philosophy and Literature, 2 (1986), pp. 304-307; M. Nussbaum, Love's Knowledge, Nueva York, Oxford University Press, 1990, pp. 5-8.

11. M. Weber. «Excurso. Teora de los estadios y direcciones del rechazo religioso del mundo*. en Ensayos sobre Sociologia de la Religión, Madrid, Taurus, 1983, vol. I, pp, 437-466, Sobre la diferenciación de esferas, J.M. Feny, «Fait, Sens, Validité dans l'architectonique des sciences contemporaines*. Revte de Metaphisique et Morale, 93 (1988), pp. 105-125.
12. Asf lo plantean S. Darwall, A, Gibbard y $\mathbf{P}$. Railton, Toward "Fin de siecle" Ethics: Some Trends*, The Philosophical Review, 101 (1992).

13. Como lo denomina J. Nancy, en J.F. Courtine y M. Deguy, Du sublime, Berlín, 1988, pp. 7-9.

14. R. Barthes, Critique et verité, Paris, Seuil, 1966, pp. 37-40; Essais critiques. Paris, Seuil, 1964, pp. 13-14, 139-142, 221-237.

15. En los términos de M. Walser, «Alpen-Laokoon oder Uber die Grenze zwischen Literatur und Gebirgew, en Wer ist ein Schrifsteller?, Francfort, Suhrkamp, 1979, p. 60.

16. M. Foucault, Histoire de la sexualite, L'usa. ge des plaisirs, Paris, Gallimard, 1984, pp. 15-19.

\title{
Composición: Adorno y el lenguaje de la filosofía
}

\author{
GERARD VILAR \\ Universidad Autónoma de Barcelona
}

Analog [zur Musik] hâtte Philosophie nicht sich auf Kategorien zu bringen sondern in gewissom Sinn erst $z L$ komponieren.

\section{Negative Dialektik, p. 44}

Al contrario de lo que ha ocurrido con otros de los nombres que se asocian a la primera generación de la Escuela de Francfort, el de T.W. Adorno no ha dejado de crecer en estatura filosófica desde quc hace ahora veinticinco años falleciera durante sus vacaciones estivales en Suiza. Ello es con seguridad indice de que una parte sustantiva de las reflexiones encerradas en su obra conectan con problemas medulares de la filosofía contemporánea. Uno de ellos es sin lugar a dudas el que, en multitud de variaciones, reproduce la clásica cuestion de a qué género discursivo pertenece la filosofía, o de la relación existente o las relaciones posibles en ella entre la lógica y la retórica. Desde modernas posiciones desconstruccionistas, postestructuralistas o neo- pragmatistas se defienden planteamientos que implican una amuerte retórica" de la filosofía en la estela del gesto anticipado ya por Nietzsche $y$ cuyo más inmediato precedente encontramos en el último Heidegger. Dicha muerte retórica de la filosofia y su zozobra en la literatura se produce como consecuencia del abandono por «metafísica» de la pretensión de verdad y racionalidad que ha acompañado al pensamiento filosófico desde la antigüedad. Derrida, Deleuze o Rorty ya no distinguen entre Hegel y Mallarmé o entre Proust y Wittgenstein más que por la fuerza literariamente iluminadora y retóricamente reveladora y patentizadora que poseen sus textos. $\mathrm{O}$, al menos, eso es lo que se sostiene acerca de la posición de dichos pensadores desde los planteamientos situados al otro extremo del espectro como los de Habermas, Putnam a Apel. En mi opinión, y sin perjuicio de las evidentes diferencias de presupuestos de unos y 